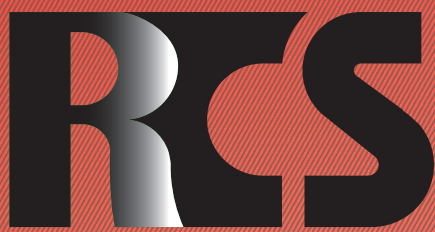

Depósito legal ppi $201502 Z U 4662$

Esta publicación científica en formato digital es continuidad de la revista impresa Depósito Legal: pp $197402 Z$ Z789

- ISSN: 1315-9518 • ISSN-E: 2477-9431

Revista de Ciencias Sociales

Universidad del Zulia. Revista de la Facultad de Ciencias Económicas y Sociales Vol. XXVII,

No. 4,2021

Esta publicación científica en formato digital es continuidad de la revista impresa Depósito Legal: pp $197402 Z 1789$ ISSN: $1315-9518$ 


\title{
Principios del liderazgo ético en directivos de la Universidad de La Guajira, Colombia
}

\author{
Pinto Aragón, Elvis* \\ Mendoza Cataño, Carmenza** \\ Villa Navas, Ana Rita***
}

\section{Resumen}

En los últimos tiempos, las exigencias del entorno han configurado una nueva identidad de universidad, más abierta, flexible, sostenible y cambiante en un marco global tecnológico y científico emergente. Dicho contexto, requiere de un liderazgo centrado en valores y con sentido de identidad, apuntando hacia una responsabilidad ética. De esta manera, el objetivo de la investigación fue analizar el liderazgo ético en directivos de la Universidad de la Guajira, Colombia. Se utilizó un estudio de tipo descriptivo, con diseño no experimental, transeccional, de campo. Los datos se recabaron mediante un cuestionario de 12 ítems, de escala frecuencial, con cinco alternativas de respuesta: Siempre, casi siempre, a veces, casi nunca y nunca, dirigido a la población conformada por cincuenta (50) directivos de la Universidad de La Guajira. Los hallazgos indican que están presentes los principios de respeto, sentido de comunidad, la justicia y equidad, honestidad y el altruismo. Se concluye, que el liderazgo ético en los directores de la Universidad de La Guajira, busca fortalecer principios con valores para promover cambios en el pensamiento organizacional, que induzcan a lograr una educación de calidad.

Palabras clave: Liderazgo ético; principios; valores; directivos; Universidad de La Guajira-Colombia.

\footnotetext{
* Magister en Gerencia de Recursos Humanos. Licenciada en Trabajo Social. Docente en la Universidad de La Guajira, Riohacha, Colombia. E-mail: epinto@uniguajira.edu.co (iD ORCID: https://orcid. org/0000-0002-9866-9751

** PhD. en Gerencia de la Educación Superior. Doctora en Ciencias Gerenciales. Magister en Gerencia de Recursos Humanos. Psicóloga. Docente en la Universidad de La Guajira, Riohacha, Colombia. E-mail: cmendoza@uniguajira.edu.co (iD) ORCID: https://orcid.org/0000-0002-7065-8794

*** Magister en Gerencia de Recursos Humanos. Psicologa. Docente en la Universidad de La Guajira, Maicao, Colombia. E-mail: anarita@uniguajira.edu.co (iD ORCID: https://orcid.org/0000-0003-2761$\underline{2448}$
}

Recibido: 2021-06-03 · Aceptado: 2021-08-21 


\title{
Principles of ethical leadership in executives of the University of La Guajira, Colombia
}

\begin{abstract}
In recent times, the demands of the environment have configured a new university identity, more open, flexible, sustainable and changing in an emerging global technological and scientific framework. Said context requires a leadership focused on values and with a sense of identity, pointing towards an ethical responsibility. In this way, the objective of the research was to analyze the ethical leadership in executives of the University of La Guajira, Colombia. A descriptive study was used, with a non-experimental, transectional, field design. The data were collected through a 12 -item questionnaire, with a frequency scale, with five response alternatives: Always, almost always, sometimes, almost never and never, addressed to the population made up of fifty (50) directors of the University of La Guajira. The findings indicate that the principles of respect, sense of community, justice and fairness, honesty and altruism are present. It is concluded that ethical leadership in the directors of the University of La Guajira, seeks to strengthen principles with values to promote changes in organizational thinking, which lead to achieving a quality education.
\end{abstract}

Keywords: Ethical leadership; principles; values; managers; Universidad de La Guajira-Colombia.

\section{Introducción}

Las organizaciones en la práctica de sus acciones, se sustentan en un liderazgo que las orienta a tomar las decisiones más asertivas y efectivas con ideas creativas, progresivas e innovadoras, adecuándose a los requerimientos y oportunidades presentes en el entorno globalizado. Así pues, ser líder demanda tener una competencia conductual que facilite articular las capacidades internas para dar respuestas a los objetivos establecidos, en una aldea global cada vez más comprometida con comportamientos éticos.

En este orden de ideas, el liderazgo se concibe como el acompañamiento, guía, direccionamiento, que una persona en la organización asume con relación a sus colaboradores interesados en que se alcancen las metas establecidas. Su importancia se hace visible, cuando se logra el compromiso de los colaboradores, inspirando confianza, dando sentido a su trabajo, y motivándolos a seguir sus objetivos (Paz, Sánchez y Sánchez, 2017; Parra, Rocha y Durán, 2021). Al respecto, Lussier y Achua (2011); Perozo-Ramírez y Paz-Marcano (2016); así como Alvear et al. (2019), refieren que el liderazgo es el proceso de influencia de líderes y seguidores para alcanzar los objetivos de la organización mediante el cambio.

Guaiquirima y Seijo (2010); Ferrer, Colmenares y Clemenza (2010); Eisenbeiss (2012); y, Quijada et al. (2017), destacan el liderazgo ético, refiriéndose a aquel que ejerce desde y hacia las personas, conformado por la confianza, credibilidad y coherencia, a fin que las actividades que se ejecutan diariamente sean las más idóneas, correctas, ubicándose dentro de la sociedad como un ente responsable.

Con base a lo anterior, el líder basado en un accionar ético, lo conlleva a actuar bajo un patrón axiológico, donde los valores como la libertad son tomados en cuenta dentro de su criterio personal, porque de nada sirve una libertad que conlleve a la persona a su propia destrucción, si no es edificada y construida desde el contexto de la vida real. Por tanto, los valores y principios, sirven de referencia para guiar y orientar la actividad humana, por ende, su actuación dentro de las organizaciones 
académicas, pues, no solo es reproducir el patrón de formación del individuo en el ámbito educativo, sino también su vinculación en la sociedad (Duran et al., 2015; Pinto, Brito y Mendoza, 2017).

Ahora bien, en la Universidad de La Guajira el liderazgo ético juega un papel esencial, porque es proyectado hacia entornos humanizados, donde intervienen la responsabilidad de accionar estrategias que integren y orienten a los directivos a comprometerse con sus líderes y con el entorno donde participa, por medio de una interacción social (Pinto, Brito y Mendoza, 2016a; Paz, Pinto y García, 2020); las universidades como parte de sus planes de desarrollo, deben incluir estrategias que coadyuven a potenciar las competencias profesionales y el bienestar general de su comunidad institucional.

En este orden de ideas, una organización académica donde sus colaboradores, entre ellos los directores adscritos a sus unidades funcionales, tenga un manejo de un liderazgo ético, conduce a buenas prácticas pautadas con una acción guiada en valores, haciendo que ellos portavoces de ideas, motiven a realizar sus trabajo con entusiasmo, con ganas de cooperar, y buscando el bien común de la organización, así como el perfeccionamiento en la calidad de impartir conocimientos transparentes e identidad compartida (Paz et al., 2016; Paz et al. 2019; Sanz et al. 2020).

Lo referido, motiva el desarrollo de este estudio, con el propósito de responder a la interrogante: ¿Cómo están los principios de liderazgo ético en los directivos de la Universidad de La Guajira?, definiendo como objetivo de la investigación: Analizar los principios de liderazgo ético en los directivos de dicha institución, con lo que se puede establecer su implementación, dado que, un líder de dirección en el campo de las universidades debe poseer habilidades y competencias que faciliten la adaptación a las transformaciones del entorno, con actuaciones en pro de las necesidades institucionales, con una visión prospectiva que le permitirá avanzar en la eficiencia de su gestión, con repercusiones concretas sobre el desarrollo social de su entorno.

\section{Principios del liderazgo ético}

Al momento en que una persona asume el liderazgo de una organización, debe marcar sus propias pautas, comportarse éticamente, desarrollar una visión y conducta que moldee el comportamiento de sus colaboradores, con base, en sus principios morales, siendo sensible a las opiniones de sus seguidores, solventando las diferencias y conflictos de una manera respetuosa y altruista (Rodrigues y De Souza, 2016; Pinto, Brito y Mendoza, 2016b; Quijada et al., 2017).

Por su parte, González et al. (2019), hacen referencia a los principios éticos en la gerencia participativa, enfocándolos en una dimensión de respeto, responsabilidad y transparencia en las funciones y procesos de trabajo, como una forma de fortalecer la actuación de sus colaboradores en las actividades desempeñadas. Es decir, que dichos principios orienten a las personas en su compromiso con una cultura ética, convirtiéndose en elemento de cambio que guía las acciones laborales, así como en sustento y orientación del comportamiento del hombre en la sociedad, y en su actuación laboral.

Dentro de este orden de ideas, Oñoro (2007); y, Villasmil, Romero y Socorro (2021), plantean que el desarrollo de un liderazgo basado en principios éticos, garantiza una mayor confianza de los colaboradores. Para ello, se cultivan valores como el respeto, sentido de comunidad, equidad, justicia, que le permite al líder marchar al ritmo que se van desarrollando los acontecimientos, sin forzar su desarrollo, permitiendo una visión clara de lo que se espera, y no abandonarla, aunque los acontecimientos sean adversos.

Por otra parte, Miras y Longás (2020), destacan que con relación al liderazgo ético se necesitan líderes generosos, nobles de espíritu, renuentes a ser adulados, que generen credibilidad, y de posturas que fortalezcan la moral. Líderes con una energía dirigida a la esperanza, a la serenidad, que generen 
confianza, y no cedan ante las tentaciones de tener cada día más.

Northouse (2012), refiere que para que exista un liderazgo ético dentro de una organización educativa, el líder debe tener presente cinco principios éticos básicos que conllevan a tratar bien a los demás y no solo como un medio para lograr alcanzar un fin común, como son el respeto, la equidad, justicia, altruista, honestidad, el sentido de comunidad. Por otra parte, $\mathrm{Ng}$ y Feldman, (2015), consideran un buen líder ético como aquel que sigue sus principios hacia resultados positivos creando un vínculo firme entre la ética y el liderazgo, y no como aquellos que ejercen un liderazgo imponiendo malas intenciones.

Por tanto, es primordial que el líder sea capaz de llevar a sus seguidores a un cambio, donde todos los actores puedan desarrollarse dentro de un ámbito donde imperen los valores, como el respeto, la honestidad, la responsabilidad para hacer crecer a la organización. De esta forma, en la investigación se presenta la postura de autores quienes destacan los principios del liderazgo ético, enfocando las perspectivas que presentan Vaca (2012); y, Northouse (2012), donde establecen seis y cinco principios, respectivamente, que todo líder ético, debe tener presente a la hora de impartir sus órdenes a sus seguidores para que exista una conexión positiva. En este sentido, se describen los cinco principios éticos que debe seguir un líder según Northouse (2012), con quien la investigación, asume su postura:

a. Respeto: Todo líder debe escuchar de manera atenta lo que sus seguidores deseen expresar, así como también debe ser empático y tolerante ante aquellas opiniones que sean opuestas a su criterio, lo que quiere decir, que debe utilizar un tratamiento, donde estén presente una actitud positiva, valores y creencias. Además, establecer hasta donde llegan sus posibilidades de hacer o no hacer, como base de toda convivencia en sociedad. Igualmente, reconocer en sí y en los demás sus derechos, así como virtudes con dignidad, dándoles a cada quien su valor. Esta igualdad exige un trato atento y respetuoso hacia todos. En ese sentido, el respeto se convierte en una condición de equidad y justicia, donde la convivencia pacífica se logra solo si se considera que este valor es una condición para vivir en paz con las personas en la sociedad.

b. Altruismo: Todo líder tiene el deber de servir a los demás, facilitando a otros su participación en el desarrollo de sus objetivos, un bienestar donde todo seguidor se sienta tomado en cuenta, que sepa que sus intereses están presentes, que ocupan un lugar importante. Una característica primordial en el liderazgo ético es precisamente el altruismo, porque cuando dirige a otra persona debe hacerlo de una manera desinteresada, sin pensar conseguir un beneficio unipersonal, sino al contrario, que su objetivo sea el bien común de los demás, pensar en la manera de cómo ayudar, apoyar a la organización para la consecución de sus metas establecidas sin esperar recompensa a cambio.

c. Justicia y equidad: Una regla primordial dentro del liderazgo ético, es que todo líder debe tratar a los demás por igualdad de condiciones, ser justo a la hora de tomar decisiones, preocuparse que exista dentro un ambiente con equidad y justicia, sustentado en valores morales. Dentro de este orden de ideas, la equidad es un valor que implica justicia e igualdad de oportunidades entre hombres y mujeres, respetando la pluralidad de la sociedad. Es una relación equitativa, cuando todos los participantes reciben resultados relativamente iguales de la relación. Asimismo, está vinculada con el principio de igualdad: Donde todos los individuos son iguales en todos los aspectos relevantes, y por tanto deben ser tratados igual.

d. Honestidad: El ser honesto implica decir la verdad en todo momento y situación. El líder tiene que ser abierto con sus seguidores, presentar la realidad de una forma lo más completa y plena posible. No obstante, debe existir un equilibrio entre la sinceridad y la omisión de una información donde cuya situación pueda ser contraproducente para los demás, y es allí, donde el líder debe colocar en práctica el buen juicio, la prudencia para 
dirimir en qué grado puede dar la información o simplemente no transmitirla. Ahora bien, el líder debe comprometerse a no tergiversar la información, a no manipularla para su bien, no esconder, no estar con evasivas sino al contrario, rendir cuenta de sus actuaciones, dar explicaciones que hagan sentir a sus seguidores que es confiable.

e. Sentido de comunidad: Tanto líderes como seguidores deben prestar atención a las demás cosas, a parte de sus propias metas, necesitan atender a las necesidades de la comunidad. Un líder ético es aquel que se preocupa porque todos los que se encuentren en su entorno social estén bien, dentro de un sentido amplio. Dentro de esta coyuntura, un líder debe asumir una actitud de colaborador ante la comunidad, a contribuir en crear un ambiente armónico dentro y fuera de la organización educativa, cuya actitud debe transcender en desarrollar una ética de la comprensión y del género humano, como elementos que ayuden a fomentar una sociedad compleja donde garantice su cohesión, no solo con leyes justas, sino con valores como la responsabilidad y solidaridad, teniendo presente el valor de la ética en todas sus actuaciones.

Por su parte, Vaca (2012) refiere que ser líder está en los pensamientos y en los labios de toda persona que quiere promover un liderazgo con conocimientos fundamentados, que poseen un enfoque vital de cómo liderar una organización sólida, porque reúne los adecuados principios de un correcto liderazgo. Para llegar a ser un buen líder debe enfocarse en estos seis principios que se describen a continuación:

a. Equidad: Es necesario que en un equipo de trabajo haya entendimiento y se tenga claro el concepto de justicia, un buen líder debe manejar bien la equidad, porque las decisiones que se tomen tienen que ser las más adecuadas e idóneas, siendo un ser abierto, y sobre todo honesto e imparcial con su equipo de trabajo.

b. Honestidad, integridad y confianza: Las personas que integran un equipo debe tener confianza en su líder y él mismo debe proporcionarle la información con detalles, decisiones tomadas de manera correcta, y para ello el dirigente debe generar esa confianza a su grupo de trabajo, debe ganarse la honestidad, la integridad, si cumple con estas pautas de seguro se ganará la comprensión y fe de su equipo, pero demostrando con hechos lo que se dice, y cuando se cometa un error o se tome mal una decisión, es recomendable decirlo, no buscar culpables donde no los hay o tratar de ocultarlo, es mejor ser honesto y seguir adelante.

c. Potencial: Cuando alguien es nuevo en el equipo de trabajo, es importante hacer sentir a esa persona que tiene potencial, para estar ahí, para crecer, adquirir nuevas habilidades y desarrollarlas. Puesto que los individuos miran al líder y quieren ser como él, porque es inspirador, les permite evolucionar, desarrollar a plenitud su potencialidad, no se debe limitar el crecimiento, asumiendo que van a ver cómo actúa, piensa, y cómo habla.

d. Respeto y dignidad: El respeto es difícil de ganar, pero fácil de perder, por ello, todo líder debe adoptar la regla de oro, tratar a las personas como quiere ser tratado, es decir, tratar a los individuos de su equipo con respeto y dignidad, por lo que son, por el trabajo que realizan, sin discriminación alguna, aceptar, respetar las discrepancias, diferencias de opiniones, diversidad. Solo se logra ganar el respeto si se actúa con dignidad hacia sí mismo; es recomendable, cuando un sujeto de su equipo realice un excelente trabajo recompensarlo en público, y si ha cometido errores corregirlo en privado, así ellos sabrán que si cometen errores no serán humillados públicamente.

e. Servicio: El líder debe crear la sensación de estar en familia, a su equipo, inculcarle la mentalidad de que si se gana o se fracasa es como equipo y no de manera individualizada. Un buen dirigente, no solo lidera un grupo, es defensor de su causa, alguien que apoya y respalda a otros, es también asegurarse de que se tiene el grupo de trabajo que necesita para el cumplimiento de sus funciones.

f. Calidad: El líder debe establecer una 
cultura que vaya más allá de las expectativas, hacer lo mejor, porque las personas buscan hacer las cosas lo mejor posible, y esforzarse para que el trabajo el cual está desempeñando sea de muy buena calidad para todos y por ende traiga mejores beneficios.

Desde esta misma tónica, las habilidades, principios, que ostentan los lideres directivos pueden ser innatas, pero también representan cualidades adquiridas en la formación académica y la experiencia práctica. Las mismas se basan en la capacidad para gestionar los recursos con eficiencia, unificando criterios y voluntades en la consecución de las metas establecidas. Por tratarse de una labor compleja, es evidente que la directiva educativa debe ser un profesional multidisciplinario, capaz de aplicar los fundamentos de la administración, la psicología y la pedagogía a situaciones concretas que se evidencian en su plantel.

En consecuencia, el directivo de una organización debe ser un líder que posea un conjunto de habilidades interdisciplinarias, que le permitan influir positivamente en la comunidad educativa, a fin de conseguir los objetivos académicos, sociales y administrativos que se ha planteado la universidad. Por tanto, el desarrollo de estas habilidades le permitirá unificar esfuerzos, cohesionar voluntades e institucionalizar un clima proactivo y por ende una mejor fluidez en la comunicación.

\section{Metodología}

En la investigación se estableció, de acuerdo con De Pelekais et al. (2012); y, Arias (2016), una tipología descriptiva con diseño no experimental, transversal y de campo, con la intención de describir las condiciones del liderazgo ético y sus principios, recolectando la información sin intentar influir o modificar percepciones, en un momento único y aplicando un instrumento de medición.

En cuanto al universo de estudio, estuvo representado por los directivos de instancias académicas y administrativas de la Universidad de La Guajira, en Riohacha,
Colombia, totalizando 50 personas. Se tomó, a todos los elementos que conforman la población, considerando que la misma es de pequeño tamaño, por lo que se estableció un censo poblacional, tal como señala Tamayo (2012), quien manifiesta que en los censos se consideran todos los elementos de la población, siendo representativa y accesible.

Con respecto al instrumento, se basó en el enfoque positivista, utilizando el método de observación mediante encuesta, por lo que se elaboró un cuestionario tipo escala frecuencial, compuesto por doce (12) items estructurados a partir de cinco (5) alternativas de respuestas como son: Siempre; casi siempre; algunas veces, casi nunca y nunca, a las que se les asignó una puntuación de cinco (5), cuatro (4), tres (3), dos (2), y uno (1), respectivamente.

De otra parte, los resultados de la validación del instrumento, indican que el mismo es altamente confiable, por lo que fue utilizado para analizar los principios éticos en los directivos de la Universidad de La Guajira, utilizando para ello la confiabilidad por alfa de Cronbach, cuyo resultado se muestra en la Tabla 1.

\section{Tabla 1}

\section{Coeficiente Alfa de Cronbach}

\begin{tabular}{llr}
\hline $\mathrm{r}_{\mathrm{tt}}=$ & Coeficiente de Alpha Cronbach & \\
\hline $\mathrm{k}=$ & Número de ítems & 16 \\
$\sum_{\substack{\mathrm{i} e \mathrm{i} \\
\mathrm{S}}}{ }^{2}=$ & Suma de varianzas de cada & 16,6 \\
$\mathrm{~S}_{\mathrm{t}}^{2}=$ & Varianza de puntajes totales & \\
$\quad$ Confiabilidad Alta & $\mathbf{r t t}=$ & $\mathbf{0 , 8 4 0}$ \\
\hline
\end{tabular}

Fuente: Elaboración propia, 2021.

En cuanto a la técnica de análisis de datos, en el estudio se utilizó la estadística descriptiva, mediante la tabla de distribución de frecuencias absoluta y relativa, así como medias, apoyándose en un Baremo de interpretación, tal como se presenta en la Tabla 2. 
Tabla 2

Baremo de interpretación de datos promedios

\begin{tabular}{ll}
\hline Rangos & \multicolumn{1}{c}{ Categorías } \\
\hline $1,00 \geq 1,80$ & Muy baja \\
$1,81 \geq 2,60$ & Baja \\
$\mathbf{2 , 6 1} \geq \mathbf{3 , 4 0}$ & Moderada \\
$\mathbf{3 , 4 1} \geq \mathbf{4 , 2 0}$ & Alta \\
$\mathbf{4 , 2 1} \geq \mathbf{5 , 0 0}$ & Muy alta \\
\hline
\end{tabular}

\section{Resultados y discusión}

Los resultados que se presentan en la Tabla 3, corresponden a las respuestas emitidas en el cuestionario, por los cincuenta directivos de la Universidad de La Guajira en Colombia.

Fuente: Elaboración propia, 2021.

Tabla 3

Principios de liderazgo ético

\begin{tabular}{|c|c|c|c|c|c|c|c|c|c|c|c|c|}
\hline \multirow{3}{*}{ Indicador } & \multicolumn{12}{|c|}{ Alternativas } \\
\hline & \multicolumn{2}{|c|}{ Siempre } & \multicolumn{2}{|c|}{ Casi Siempre } & \multicolumn{2}{|c|}{ A veces } & \multicolumn{2}{|c|}{ Casi Nunca } & \multicolumn{2}{|c|}{ Nunca } & \multirow{2}{*}{ Total } & \multirow{2}{*}{ Media } \\
\hline & $\mathbf{F a}$ & Fr & Fa & $\mathbf{F r}$ & $\mathbf{F a}$ & Fr & $\mathbf{F a}$ & $\mathbf{F r}$ & $\mathbf{F a}$ & $\mathbf{F r}$ & & \\
\hline Respeto & 30 & $60 \%$ & 4 & $8 \%$ & 11 & $22 \%$ & 3 & $6 \%$ & 2 & $4 \%$ & 50 & 4,03 \\
\hline $\begin{array}{l}\text { Sentido de } \\
\text { Comunidad } \\
\end{array}$ & 35 & $70 \%$ & 0 & $0 \%$ & 13 & $26 \%$ & 0 & $0 \%$ & 2 & $4 \%$ & 50 & 4,26 \\
\hline $\begin{array}{r}\text { Justicia y } \\
\text { Equidad } \\
\end{array}$ & 36 & $72 \%$ & 5 & $10 \%$ & 6 & $12 \%$ & 3 & $6 \%$ & 0 & $0 \%$ & 50 & 4,48 \\
\hline Honestidad & 44 & $88 \%$ & 4 & $8 \%$ & 2 & $4 \%$ & 0 & $0 \%$ & 0 & $0 \%$ & 50 & 4,85 \\
\hline Altruismo & 36 & $72 \%$ & 0 & 0,0 & 5 & $10 \%$ & 0 & $0 \%$ & 9 & $18 \%$ & 50 & 4,04 \\
\hline Promedio & 36 & $72 \%$ & 2 & $4 \%$ & 7 & $14 \%$ & 2 & $4 \%$ & 3 & $6 \%$ & 50 & 4,33 \\
\hline
\end{tabular}

Fuente: Elaboración propia, 2021.

En la Tabla 3, en lo referente al indicador respeto, se observó que un $60 \%$ de los encuestados afirma que siempre respeta las diferencias de opinión dentro de su grupo de trabajo, un $22 \%$ dijo que a veces, un $8 \%$ casi siempre, mientras que un $6 \%$ se refirieron a las opciones casi nunca, y $4 \%$ nunca. Con respecto a la media aritmética, ésta se ubicó en 4,03, lo cual, se sitúa en el rango de interpretación de "Alto", corroborándose de esta manera que los directivos encuestados de la Universidad de La Guajira consideran el respeto hacia los demás.
Esta perspectiva coincide con lo teorizado por Northouse (2012); y, Villasmil et al. (2021), donde refieren que todo líder debe escuchar y respetar las opiniones de las personas, siendo empático y tolerante ante aquellas opiniones que vayan opuestas a su criterio, utilizando un tratamiento donde siempre este presente una actitud positiva de valores y creencias.

En atención al indicador sentido de comunidad, se observó en la Tabla 3, que el $70 \%$ de los directivos estima que siempre, 
plantean propuestas que vayan destinadas al desarrollo de actividades innovadoras dentro de la comunidad universitaria, el $26 \%$ respondió que a veces, mientras que un $4 \%$ nunca. Dentro de este marco, la media aritmética resultante fue de 4,26 la cual, según el baremo establecido tiene categoría de "Muy alto", de lo cual se deduce que los directivos, a pesar que reaccionan de forma agresiva cuando sus indicaciones no son acatadas por la comunidad universitaria, siempre plantean propuestas innovadoras dirigidas a dicha comunidad.

Lo señalado, guarda sintonía con lo referido por Northouse (2012); y, García, Paz y Cardeño (2018), quienes acotan que todo líder debe atender a las necesidades que presente la comunidad, que se preocupe que todos sus seguidores se encuentren bien dentro de un sentido amplio, asumiendo una actitud de colaborador, creando un ambiente armónico dentro de la comunidad universitaria, donde se garanticen leyes justas, acompañadas con valores tales como el respeto, la solidaridad y responsabilidad en todas sus actuaciones.

En este mismo orden de ideas, en cuanto al indicador justicia y equidad, se constató que, por un lado, el $72 \%$ de los encuestados manifestaron que siempre presentan un comportamiento ético dentro de la universidad (ver Tabla 3), y que sus decisiones son argumentadas coherentemente frente a una pluralidad de opiniones; por otro lado, 10\% mencionó que casi siempre, a veces un $12 \%$ y casi nunca $6 \%$.

Desde el punto de vista del análisis a través de la media aritmética, se obtuvo un resultado de 4,48 con categorización de "Muy alto", lo cual indica que los directivos de la Universidad de La Guajira, se caracterizan por poseer un comportamiento ético y profesional dentro de la universidad, así como también sus decisiones son tomadas coherentemente. Como se evidencia, las anteriores consideraciones guardan completa relación con lo sostenido por Northouse (2012); así como Miras y Longás (2020), que refieren que los líderes deben tratar a todos en igualdad de condiciones. Asimismo, la toma de decisiones debe hacerlo con justicia y equidad fundamentadas en motivos claros y tener presente los valores morales.

Dentro de este mismo orden de ideas, los resultados del cuestionario para el indicador honestidad, indican que el $88 \%$ de los directivos siempre reconoce los logros profesionales obtenidos por sus subordinados, y que ha observado comportamientos deshonestos dentro de la universidad, mientras 8\% afirmó casi siempre, y un $4 \%$ a veces (ver Tabla 3 ). En tal sentido, la media aritmética arrojó un valor de 4,85 con categoría de acuerdo al baremo de interpretación de "Muy alto", lo cual indica que sí existen comportamientos deshonestos pero que también se reconocen los logros profesionales obtenidos por los subordinados, por parte de los directivos de la universidad.

En función de este resultado, se infiere relación con lo expresado por Northouse (2012), cuando infiere que en todo líder debe haber un equilibrio entre la sinceridad y la omisión de una información, donde la situación pueda ser contraproducente para los demás, y es precisamente ahí donde el líder ético debe practicar su buen juicio, la prudencia, con el fin que pueda visualizar en qué grado puede dar o no, la información.

Para el indicador altruismo, se aprecia en la Tabla 3, que $72 \%$ de los encuestados respondió que siempre existe una orientación efectiva que hace que los subordinados alcancen las metas propuestas, así como también realizan actividades destinadas a potencializar a los subordinados, en tanto que $18 \%$ manifestó que nunca, y un $10 \%$ a veces. La media aritmética para este indicador se postuló en 4,04, considerada "Alta", indicando que en la Universidad de La Guajira los líderes son altruistas y buscan siempre que sus subordinados alcancen y potencialicen sus metas por medio de actividades encaminadas al desarrollo de su potencialidad.

Lo expresado, coincide con lo señalado por Northouse (2012); y, González et al. (2019), cuando afirman que el altruismo es una condición vinculada al liderazgo ético, donde quien dirige procesos organizacionales pretende el bien común de los demás, pensando en ayudar, apoyar, para la consecución de sus 
metas establecidas sin esperar recompensa a cambio.

\section{Conclusiones}

Los resultados obtenidos permitieron evidenciar que, en los directivos de la Universidad de La Guajira están presentes cinco principios éticos, siendo éstos el respeto; la honestidad; justicia y equidad; el altruismo; y el sentido de comunidad. Lo que se considera muy significativo, desde la perspectiva de un liderazgo ético. Concluyendo por tanto que, los directivos se ocupan en valorar el respeto a la diferencia y opinión de otros colaboradores, generando climas de oportunidades, mayor compromiso futuro, así como sentido de responsabilidad.

Asimismo, se enfocan en reconocer los logros alcanzados por el personal docente y administrativo, asumiendo comportamientos de orientadores colaborativos. Adicionalmente, plantean propuestas destinadas al desarrollo de actividades innovadoras dentro de la comunidad universitaria, propiciando escenarios donde prevalezcan la justicia y equidad, acompañado esto por su comportamiento ético, valorando la iniciativa, así como apoyando acciones encaminadas al beneficio de todos, en forma desinteresada como parte de la filosofía institucional.

Estos resultados encontrados muestran que sí es posible construir dentro de la Universidad, un marco de principios organizacionales sustentados en un liderazgo ético, que permee no solo a los directivos sino a los otros colaboradores en los distintos niveles de la estructura, lo cual se constituye en posibles líneas de investigación a ser abordadas en futuras investigaciones, contribuyendo así a fortalecer el conocimiento científico y en dar pautas en el pensamiento organizativo, apoyando un modelo universitario adaptado a los nuevos contextos sociales, con unas características inherentes que definen su gestión integradora, así como participativa, que alberguen las bases de sus actividades misionales.

\section{Referencias bibliográficas}

Alvear, L., Luna, O., Navarro, S., y Salas, B. (2019). Estilos de liderazgo de la alta dirección en industrias exportadoras de Barranquilla. Revista Venezolana de Gerencia (RVG), 24(86), 575-591.

Arias, F. (2016). El proyecto de investigación. Editorial Episteme.

De Pelekais, C., Finol, M., Neuman, N., Carrasquero, E., García, J., y Leal, M. (2012). El ABC de la investigación. Un encuentro con la ciencia. AstroData

Duran, S. E., Silvera, A., Parra, M. A., y Hernández, H. G. (2015). Formación en valores como eje transversal del proyecto educativo colombiano. REVISTA THE EXPO. Semilleros \& Jóvenes Investigadores, 1(1), 53-66.

Eisenbeiss, S. A. (2012). Re-thinking ethical leadership: An interdisciplinary integrative approach. The Leadership Quarterly, 23(5), 791-808. https://doi. org/10.1016/j.leaqua.2012.03.001

Ferrer, J., Colmenares, F., y Clemenza, C. (2010). Un líder ético para el cambio: plataforma de gestión estratégica en Instituciones Universitarias. Revista de Ciencias Sociales (Ve), XVI(4), 642-653.

García, J., Paz, A., y Cardeño, E. (2018). Liderazgo ético. Una perspectiva en universidades públicas del estado Zulia. Opción, 34(86), 696-730.

González, L., Paz, A., García, J., y Cardeño, E. (2019). Principios éticos de la gerencia participativa: Modelo de gestión en las empresas mixtas petroleras venezolanas. Revista Espacios, 40(4), 16-28.

Guaiquirima, C., y Seijo, C. (2010). Liderazgo ético: Un constructo ambiguo en la administración pública. En C. Seijo 
(Comp.), La gerencia en la sociedad. Un camino para la construcción de organizaciones futuras con rostro humano (pp. 57-73). Editorial Astro Data.

Lussier, R. N., y Achua, C. F. (2011). Liderazgo: Teoría, aplicación y desarrollo de habilidades. Cengage Learning Editores.

Miras, J., y Longás, J. (2020). Liderazgo pedagógico y liderazgo ético: Perspectivas complementarias de la nueva dirección escolar. Revista de Estudios y Experiencias en Educación, 19(41), 287-305. https://dx.doi. org/10.21703/rexe.20201941miras16

Ng, T. W. H., y Feldman, D. (2015). Ethical leadership: Meta-analytic evidence of criterion-related and incremental validity. Journal of Applied Psychology, 100(3), 948-965. https:// doi.org/10.1037/a0038246

Northouse, P. G. (2012). Leadership: Theory and practice. SAGE Publications, Inc.

Oñoro, R. C. (2007). Ensayos de gerencia social otra gerencia es posible. Eumed.net. https://www.eumed.net/ libros-gratis $/ 2007 \mathrm{c} / 315 /$

Parra, M., Rocha, G., y Durán, S. (2021). Liderazgo como prospectiva del clima organizacional en el sector hotelero. Revista de Ciencias Sociales (Ve), XXVII(2), 217-227. https://doi. org/10.31876/rcs.v27i2.35908

Paz, A., Núñez, M. G., García, J., y Salom, J. (2016). Rol del liderazgo ético en organizaciones académicas. Opción, 32(12), 148-168.

Paz, A., Pinto, E., Cantillo, N., García, J., y Suarez, H. (2019). Liderazgo femenino: Un estilo de gestión en la Universidad de La Guajira (Colombia). Revista Espacios, 40(37), 2-15.

Paz, A., Pinto, E., y García, J. (2020).
Universidad y sociedad aliado de la actividad extensionista para el desarrollo del emprendimiento en Venezuela. Aglala, 11(1), 47-63. https://revistas.curn.edu.co/index.php/ aglala/article/view/1569

Paz, A., Sánchez, J., y Sánchez, I. (2017). Capital Humano en las empresas distribuidoras de agua domiciliaria del departamento de la Guajira. Editorial de la Universidad de La Guajira.

Perozo-Ramírez, B. A., y Paz-Marcano, A. I. (2016). Estilos de liderazgo femenino como factor influyente en las habilidades de los equipos de trabajo en el sector asegurador. Clio América, 10(19), 8-22. https://doi. org/10.21676/23897848.1656

Pinto, E., Brito, C., y Mendoza, C. (2016a). Calidad de vida filosofía laboral de la Universidad de La Guajira. Editorial Universidad de La Guajira.

Pinto, E., Brito, C., y Mendoza, C. (2016b). Equidad económica dimensión de la calidad de vida laboral en la universidad de La Guajira. Desarrollo Gerencial, 8(2), 124-143. https://doi. org/10.17081/dege.8.2.2566

Pinto, E., Brito, C., y Mendoza, C. (2017). Ética y moral en el contexto de las Organizaciones. Editorial Universidad de La Guajira.

Quijada, G., Pulgar, C., Prieto, R., y Rincón, Y. (2017). Inteligencia ética: Un enfoque teórico hacia el liderazgo. En R. Prieto y A. M. Cazallo (Comps.), Desarrollo organizacional y gestión humana en contextos globalizados (pp. 269-310). Ediciones Universidad Simón Bolívar.

Rodrigues, J., y De Souza, F. (2016). Ethical leadership, leader-follower relationship and performance: A study in a telecommunications company. RAM. Revista Administração 
Mackenzie, 17(2), 67-92. https:// doi.org/10.1590/1678-69712016/ administracao.v17n2p67-92

Sanz, D. A., García, J. E., Prieto, R. A., y Paz, A. I. (2020). Lineamientos estratégicos para fortalecer la gestión gerencial en universidades de la costa atlántica. Revista de Ciencias Sociales (Ve), XXVI(E-2), 155-171. https:// doi.org/10.31876/rcs.v26i0.34120

Tamayo, M. (2012). El proceso de la investigación científica. Editorial

\section{Limusa.}

Vaca, G. R. (2012). Como ser un buen lider: Principios de liderazgo que cambiarán tu vida para siempre. Editorial Palibrio.

Villasmil, M. D C., Romero, F. J., y Socorro, C. C. (2021). Liderazgo ético en la gestión pública municipal del estado Zulia, Venezuela. Revista de Ciencias Sociales (Ve), XXVII(2), 199-216. https://doi.org/10.31876/rcs. v27i2.35907 